\title{
Hematological, Biochemical and Biometric Changes in Clarias Gariepinus Exposed to Antipsychotic Drug Chlorpromazine
}

\section{Martins Nnamdi Okpe}

Enugu State University of Science and Technology

Christabel Chukwuebuka Eze

Enugu State University of Science and Technology

Hope Chinwe Ezinwa

University of Nigeria Faculty of Biological Sciences

\section{Uduak Aletan}

National Open University of Nigeria

Henrietta ljeoma Kelle

National Open University of Nigeria

Maureen N Chukwu

National Open University of Nigeria

Martin Abdubala Okpanachi

Kogi State University

Oscar Ifeanyi Aguzie

University of Nigeria Faculty of Biological Sciences

Christopher Didigwu Nwani ( $\nabla$ chris.nwani@unn.edu.ng )

University of Nigeria Faculty of Biological Sciences https://orcid.org/0000-0003-4628-1621

\section{Research Article}

Keywords: Chlorpromazine, behavior, biochemistry, hematology, morphological parameters, fish

Posted Date: June 16th, 2021

DOl: https://doi.org/10.21203/rs.3.rs-560297/v1

License: (c) (i) This work is licensed under a Creative Commons Attribution 4.0 International License. Read Full License 


\section{Abstract}

Chlorpromazine (CPZ) is a neuroleptic and antipsychotic medication for individuals suffering from schizophrenia and other medical conditions. This study investigated the effects of CPZ on the hematological, biochemical, and biometric characteristics in juvenile Clarias gariepinus. The fish were exposed to $0.53,1.06$ and $2.11 \mathrm{mgl}^{-1} \mathrm{CPZ}$ for 15 days after which they were withdrawn from the toxicant and allowed to recover for 5 days. Blood were sampled from the fish on day 1, 5, 10, 15 and during the 5day recovering for hematological and biochemical analysis and thereafter, the fish were sacrificed for the morphometric analysis. While the values of the white blood cells significantly increased in the exposed fish, the hemoglobin, erythrocytes and packed cell volume decreased. Compared with the control, there were no significant differences in the values of the blood derivatives in the exposed fish. The values of protein and glucose reduced but that of aspartate aminotransferase, alanine aminotransferase and alkaline phosphatase were significantly elevated. Though there was no significant difference in the condition factor, a significant increase in hepatosomatic index occurred on day-15 at $5.28 \mathrm{mg} / \mathrm{L} \mathrm{CPZ}$. After the 5-day withdrawal from the drug, most of the studied parameters returned to the control values. The present study indicated that CPZ is toxic to fish and should be used with utmost care to guard against toxicological effect on non-target organisms.

\section{Introduction}

Chlorpromazine (CPZ) is a drug popularly used for the treatment of psychotic challenges in humans. It is also used in treatment of children with behavioral and attention disorders. The mechanism of action of the drug is the blockage of the dopamine receptors in the central nervous system such as the brain (Susuki et al., 2013). This in turn lowers the accumulation of excess dopamine in the brain thus leading to various degrees of suppression of the psychotic symptoms.

The high level of pharmaceuticals and personal care products in the aquatic environments reported recently calls for concern due to the eco-toxicological effects on non-target organisms (Ebele 2020). Studies indicate that CPZ was harmful to aquatic micro algal species (Porsbring et al., 2008), Daphnia magna (Oliveira et al., 2016) and Carassius auratus (Li et al., 2008). It has also suppressed steroidogenesis in organisms (Hinfray et al., 2011; Wassmur et al., 2013). In a related report, CPZ elevated brain acetylcholinesterase values, lipid peroxidation and caused variations in the values of antioxidant enzymes in African catfish Clarias gariepinus (Atama et al., 2020). Further, pharmaceuticals and personal care products residues in the aquatic system can cause irreversible changes in the hematological, biochemical and processes in the organisms. Chlorpromazine residues of about 5 to $364 \mathrm{ng} \mathrm{I}^{-1}$ was detected in effluents from waste water treatment plant (Yuan et al.,2013) while concentrations up to 0.9 to $2.6 \mathrm{ng} \mathrm{I}^{-1}$ had been detected in surface water in Spain (Fernandez et al., 2010). Though the presence of $\mathrm{CPZ}$ in various water bodies have been reported, information regarding the hematological, biochemical and biometric profile of $C$. gariepinus after exposure to $\mathrm{CPZ}$ are scarce. The African Catfish $C$. gariepinus is one the commercial indigenous fish species preferred due to its fast growth, nutritional quality, easy domestication, high reproductive potential and adaptability to extreme water quality condition. The aim 
of the present study was to investigate if $\mathrm{CPZ}$ can alter the hematological, biochemical and biometric characteristics of indigenous African Catfish C. gariepinus juveniles.

\section{Materials And Methods}

\section{Procurement of fish and drug}

African catfish $C$. gariepinus juveniles $(n=300)$, mean length $30.13 \pm 0.25 \mathrm{~cm}$ and weight, $152.38 \pm 2.24 \mathrm{~g}$ were procured from Freedom Fish Farm Limited Nsukka and transported to the Fisheries Wet laboratory of our institution where they were acclimatized for two weeks in three concrete ponds using nonchlorinated tap water. They were given $35 \%$ crude protein diet three times daily. The behavioral responses of the fish such as opercula movement, erratic swimming, air gulping, mucus secretions and skin coloration were observed and recorded. Commercial pharmaceutical preparations of CPZ with the trade name Largactil (100 mg CPZ) manufactured by Oubari Pharmaceutical Ltd, Aleppo-Syria, under license from Aventis Laboratory- France was used.

\section{Design For Sublethal Exposure}

The $96 \mathrm{~h}$ median lethal concentration ( $\mathrm{LC}_{50}$ ) of $\mathrm{CPZ}$ was calculated to be $10.56 \mathrm{mg} \mathrm{I}^{-1}$ for $C$. gariepinus juveniles. Three sub-lethal CPZ concentrations viz: $2.112 \mathrm{mgl}^{-1}$ (1/5th of $\left.96 \mathrm{~h} \mathrm{LC} 50\right), 1.056 \mathrm{mgl}^{-1}(1 / 10$ th of $96 \mathrm{~h})$ and $0.528 \mathrm{mgl}^{-1}\left(1 / 20\right.$ th of $\left.96 \mathrm{hC}_{50}\right)$ were selected for the in vivo experiment. The fish were divided into four treatment groups (Groups I, II, III and IV). Group I was exposed to $0.528 \mathrm{mgl}^{-1} \mathrm{CPZ}$, Group II to $2.11 \mathrm{mgl}^{-1}$, Group III to $1.06 \mathrm{mg} \mathrm{I}^{-1}$, and Group IV which served as control to dechlorinated water only. The groups were set in replicates of three, each containing ten fish. The test solution was renewed every $48 \mathrm{~h}$ to ensure that the concentration of the drug remain the same. The level of CPZ in the aquaria was analyzed using liquid chromatography - mass spectrometry (LC-MS) (Ajima et al., 2017) and did not significantly differ from the nominal concentrations. During the sublethal experiment, no mortality was observed during the 20-days exposure. Thereafter the fish were withdrawn and kept in drug free aquarium for another 5 days for possible recovery. The fish were fed daily throughout the experiment with about $3 \%$ of total body weight an hour before renewal of the test solution to avoid mortality. The behavioral changes during the sublethal exposure and recovery were noted and recorded. The physicochemical parameters of the test water (APHA, AWWA, WPCE, 2005) were: dissolved oxygen $7.14 \mathrm{mgl}^{-1}$, temperature $27.83^{\circ} \mathrm{C}, \mathrm{pH} 12.3$ and conductivity $247.5 \mu \mathrm{Scm}^{-1}$.

\section{Blood sample collection}

Blood sample collection was made on the first day of exposure to CPZ and subsequently, on day 5,10 , 15, 20 and 25 (i.e. 5-day recovery period). About $3.0 \mathrm{~mL}$ of blood was obtained by cardiac puncture using a hypodermic heparinized syringe, and transferred into small EDTA bottle. Two fish from each replicate experiment and control were sampled on each sampling day. Every sampled fish was removed from the experimental system to avoid multiple blood collection from the same fish. One half of the blood was 
used for estimation of the hematological parameters. The remaining half was centrifuged at $10000 \mathrm{~g}$ at $4^{\circ} \mathrm{C}$ for 20 min to separate the plasma which was used for the estimation of biochemical parameters.

\section{Hematological Assays}

Red blood cells count was determined by the method of Ochei and Kolhatkar, (2008). The blood specimen was diluted 1:200 with RBC diluting fluid and cells were counted under high power (40 X) objective of a microscope by using a counting chamber. The number of cells were calculated and reported as the number of red cells / cu.mm of whole blood. The white blood cell count, PCV and hemoglobin concentration $(\mathrm{Hb})$ were determined following the method described by Ochei and Kolhatkar (2008). Erythrocyte indices, such as mean corpuscular hemoglobin concentration (MCHC), mean corpuscular hemoglobin $(\mathrm{MCH})$ and mean corpuscular volume $(\mathrm{MCV})$ were calculated from the results of RBC count, $\mathrm{Hb}$ and PCV according to standard formulae (Dacie and Lewis, 1984):

$$
\mathrm{MCV}(\mathrm{fl})=\frac{P C V(\%) \times 10}{R B C \text { count in millions } / \mathrm{mm} 3}
$$

$\mathrm{MCH}(\mathrm{pg})=\frac{\mathrm{Hb} \times\left(\begin{array}{l}g \\ d\end{array}\right) \times 10}{R B C \text { count in millions } / \mathrm{mm}^{3}}$

$\operatorname{MCHC}\left(\begin{array}{l}g \\ d l\end{array}\right)=\frac{H \mathrm{~b} \times\left(\begin{array}{c}g \\ d V\end{array}\right) \times 100}{P C V(\%)}$

\section{Estimation Of Biochemical Indices}

Randox diagnostic kits were used for biochemical tests. The activity of aspartate aminotransferase was assayed by the method of Reitman and Frankel (1957) as outlined in the Randox kit. Activities of alkaline phosphatase was assayed as outlined in the kit. The protein and glucose level was determined using Randox Kit as described by (Reitman and Frankel, 1957).

\section{Morphometric Variations}

During each sampling period the standard length and body weight of the fish were determined. The liver was excised and weight also measured. Following the methods of White and Fletcher (1985), the condition factor (CF) and hepatosomatic index ( $\mathrm{HSI})$ were calculated as:

$\mathrm{CF}=$ Body weight $(\mathrm{g}) /$ Standard length $(\mathrm{cm})^{3} \times 100$

HSI = Liver weight $(\mathrm{g}) /$ Body weight $(\mathrm{g}) \times 100$

\section{Statistical analysis}

The statistical packages for social sciences (SPSS) version 23.0 (IBM Corporation, Armonk, New York) was used to analyze the data obtained. Two-way analysis of variance with CPZ concentration and 
exposure duration as fixed factors and biochemical and hematological parameters as dependent factors were used. Post-hoc comparison was by LSD and p-values adjusted by the Bonferroni method. Values were presented as mean \pm standard deviation, and level of significance set at $p<0.05$. Effect size reported was partial eta squared $\left(n_{p}{ }^{2}\right)$.

\section{Results}

\section{Changes in behavior in C. gariepinus exposed to CPZ}

There were changes in behavioral responses such as loss of balance, skin coloration, abnormal mucus secretion, swimming rate, opercula movement and air gulping during sublethal exposure and recovery of C. gariepinus to CPZ (Table 1). The skin coloration, mucus secretion and air gulping increased with increased concentrations of CPZ while swimming rate and opercula movement decreased. After 5-days recovery, the skin coloration, mucus secretion and air gulping were reduced while the swimming rate and opercula movement became stable.

Table 1

Changes in behavior of Clarias gariepinus after 10 - days exposure to sublethal concentrations of chlorpromazine and 5 - days recovery.

\begin{tabular}{|llllll|}
\hline & & \multicolumn{5}{c|}{ Chlorpromazine concentrations (mg/L) } \\
\hline Exposure duration & Parameter & Control & 1.06 & 2.11 & 5.28 \\
\hline 15 - days & Skin coloration & - & ++ & ++ & +++ \\
\hline & Mucus secretion & - & ++ & ++ & +++ \\
\hline & Swimming rate & - & ++ & ++ & + \\
\hline & Opercula movement & - & ++ & + & + \\
\hline & Air gulping & - & + & ++ & ++ \\
\hline 5-days recovery & Skin coloration & - & ++ & ++ & + \\
\hline & Mucus secretion & - & ++ & ++ & + \\
\hline & Swimming rate & - & + & + & + \\
\hline & Opercula movement & - & + & + & + \\
\hline & Air gulping & - & + & - & - \\
\hline (-) Indicates normal behavior, (+) mild, (++) moderate and (+++) high. & \\
\hline
\end{tabular}

Hematological changes in C. gariepinus exposed to CPZ 
Chlorpromazine exposure was associated with reduction in PCV, RBC, $\mathrm{Hb}$ and increased WBC (Table 2). $\operatorname{PCV}\left(F_{3,40}=56.505, \mathrm{p}<0.0001, \eta_{\mathrm{p}}{ }^{2}=0.809\right), \operatorname{RBC}\left(\mathrm{F}_{3,40}=76.935, \mathrm{p}<0.0001, \eta_{\mathrm{p}}{ }^{2}=0.852\right)$ and $\mathrm{Hb}\left(\mathrm{F}_{3,40}=\right.$ $\left.129.704, p<0.0001, \eta_{p}^{2}=0.907\right)$ decreased significantly from the drug exposure. The decrease in all three parameters were duration-dependent $\left(F_{4,40}=19.188,52.279,69.256, p<0.0001\right.$ respectively), and most pronounced on day-15. Leucocytosis induced by the drug was significant $\left(F_{3,40}=185.543, p<0.0001, \eta_{p}{ }^{2}\right.$ $=0.933)$, and similarly duration-dependent $\left(F_{3,40}=124.674, p<0.0001, \eta_{p}{ }^{2}=0.926\right)$, effect noticed from day-5 and continued till 5-day post withdrawal. The values of the neutrophil, basophil, eosinophil, lymphocytes and monocytes were not affected by CPZ (supplementary Table S1). 
Table 2

Changes in packed cell volume (PCV), red blood cell count (RBC), haemoglobin concentration (Hb), and white blood cell count (WBC) on 15-day exposure of juvenile Clarias gariepinus to CBZ

\begin{tabular}{|c|c|c|c|c|c|c|}
\hline \multirow[t]{2}{*}{ Parameter } & \multirow{2}{*}{$\begin{array}{l}\text { Conc. } \\
\text { (mg/L) }\end{array}$} & \multicolumn{5}{|c|}{ Duration (Day) } \\
\hline & & 1 & 5 & 10 & 15 & 5-d with. \\
\hline \multirow[t]{4}{*}{ PCV (\%) } & Control & $\begin{array}{l}27.00 \pm \\
2.65^{\mathrm{a} 1}\end{array}$ & $\begin{array}{l}32.33 \pm \\
4.04^{\mathrm{a} 1}\end{array}$ & $\begin{array}{l}28.33 \pm \\
2.08^{\mathrm{a} 1}\end{array}$ & $\begin{array}{l}31.67 \pm \\
2.31^{\mathrm{a} 1}\end{array}$ & $\begin{array}{l}32.33 \pm \\
3.79^{\mathrm{a} 1}\end{array}$ \\
\hline & 1.06 & $\begin{array}{l}26.00 \pm \\
1.00^{\mathrm{a} 2}\end{array}$ & $\begin{array}{l}22.00 \pm \\
3.46^{\mathrm{b} 12}\end{array}$ & $\begin{array}{l}17.67 \pm \\
1.53^{\mathrm{b} 1}\end{array}$ & $\begin{array}{l}18.69 \pm \\
1.54^{\mathrm{b} 1}\end{array}$ & $\begin{array}{l}24.67 \pm \\
0.59^{\mathrm{b} 2}\end{array}$ \\
\hline & 2.11 & $\begin{array}{l}27.33 \pm \\
1.16^{\mathrm{a} 2}\end{array}$ & $\begin{array}{l}21.33 \pm \\
1.18^{\mathrm{b} 1}\end{array}$ & $\begin{array}{l}21.33 \pm \\
1.10^{\mathrm{b} 1}\end{array}$ & $\begin{array}{l}16.67 \pm \\
1.16^{\mathrm{b} 1}\end{array}$ & $\begin{array}{l}25.33 \pm \\
1.07^{\mathrm{b} 2}\end{array}$ \\
\hline & 5.28 & $\begin{array}{l}27.67 \pm \\
2.08^{\mathrm{a} 2}\end{array}$ & $\begin{array}{l}21.67 \pm \\
0.58^{\mathrm{b} 1}\end{array}$ & $\begin{array}{l}20.33 \pm \\
1.17^{b 1}\end{array}$ & $\begin{array}{l}17.77 \pm \\
2.89^{\mathrm{b} 1}\end{array}$ & $\begin{array}{l}24.33 \pm \\
1.54^{\mathrm{b} 2}\end{array}$ \\
\hline \multirow[t]{4}{*}{$\begin{array}{l}\text { RBC } \\
\left(10^{12} / L\right)\end{array}$} & Control & $\begin{array}{l}8.04 \pm \\
0.56^{\mathrm{a} 1}\end{array}$ & $\begin{array}{l}8.95 \pm \\
0.44^{\mathrm{a} 1}\end{array}$ & $\begin{array}{l}8.75 \pm \\
0.47^{\mathrm{a} 1}\end{array}$ & $\begin{array}{l}8.54 \pm \\
0.98^{\mathrm{a} 1}\end{array}$ & $\begin{array}{l}8.86 \pm \\
0.61^{\mathrm{a} 1}\end{array}$ \\
\hline & 1.06 & $\begin{array}{l}8.15 \pm \\
0.28^{\mathrm{a} 2}\end{array}$ & $\begin{array}{l}5.98 \pm \\
0.68^{\mathrm{b} 1}\end{array}$ & $\begin{array}{l}6.47 \pm \\
0.77^{\mathrm{b} 1}\end{array}$ & $\begin{array}{l}5.10 \pm \\
0.40^{\mathrm{b} 1}\end{array}$ & $\begin{array}{l}7.07 \pm \\
0.18^{\mathrm{a} 2}\end{array}$ \\
\hline & 2.11 & $\begin{array}{l}7.82 \pm \\
0.75^{\mathrm{a} 2}\end{array}$ & $\begin{array}{l}5.85 \pm \\
0.17^{b 1}\end{array}$ & $\begin{array}{l}5.08 \pm \\
0.37^{\mathrm{b} 1}\end{array}$ & $\begin{array}{l}4.36 \pm \\
0.03^{b 1}\end{array}$ & $\begin{array}{l}7.95 \pm \\
0.31^{\mathrm{a} 2}\end{array}$ \\
\hline & 5.28 & $\begin{array}{l}8.46 \pm \\
0.13^{\mathrm{a} 2}\end{array}$ & $\begin{array}{l}5.48 \pm \\
0.13^{\mathrm{b} 1}\end{array}$ & $\begin{array}{l}5.83 \pm \\
0.16^{\mathrm{b} 1}\end{array}$ & $\begin{array}{l}5.21 \pm \\
0.52^{\mathrm{b} 1}\end{array}$ & $\begin{array}{l}8.13 \pm \\
0.42^{\mathrm{a} 2}\end{array}$ \\
\hline \multirow[t]{4}{*}{$\mathrm{HB}(\mathrm{g} / \mathrm{dL})$} & Control & $\begin{array}{l}6.60 \pm \\
0.17^{\mathrm{a} 1}\end{array}$ & $\begin{array}{l}8.13 \pm \\
0.38^{\mathrm{a} 1}\end{array}$ & $\begin{array}{l}8.10 \pm \\
0.70^{\mathrm{a} 1}\end{array}$ & $\begin{array}{l}8.17 \pm \\
0.81^{\mathrm{a} 1}\end{array}$ & $\begin{array}{l}8.17 \pm \\
0.93^{\mathrm{a} 1}\end{array}$ \\
\hline & 1.06 & $\begin{array}{l}6.57 \pm \\
0.25^{\mathrm{a} 2}\end{array}$ & $\begin{array}{l}5.20 \pm \\
0.17^{\mathrm{b} 1}\end{array}$ & $\begin{array}{l}4.47 \pm \\
0.46^{\mathrm{b} 1}\end{array}$ & $\begin{array}{l}4.40 \pm \\
0.20^{\mathrm{b} 1}\end{array}$ & $\begin{array}{l}7.53 \pm \\
0.21^{\mathrm{a} 2}\end{array}$ \\
\hline & 2.11 & $\begin{array}{l}6.47 \pm \\
0.15^{\mathrm{a} 2}\end{array}$ & $\begin{array}{l}5.50 \pm \\
0.20^{\mathrm{b} 2}\end{array}$ & $\begin{array}{l}4.90 \pm \\
0.26^{\mathrm{b} 1}\end{array}$ & $\begin{array}{l}4.07 \pm \\
0.12^{\mathrm{b} 1}\end{array}$ & $\begin{array}{l}7.07 \pm \\
0.21^{\mathrm{a} 2}\end{array}$ \\
\hline & 5.28 & $\begin{array}{l}6.57 \pm \\
0.15^{\mathrm{a} 2}\end{array}$ & $\begin{array}{l}5.43 \pm \\
0.38^{\mathrm{b} 1}\end{array}$ & $\begin{array}{l}4.07 \pm \\
0.12^{\mathrm{b} 1}\end{array}$ & $\begin{array}{l}4.07 \pm \\
0.12^{\mathrm{b} 1}\end{array}$ & $\begin{array}{l}7.13 \pm \\
0.21^{\mathrm{a} 2}\end{array}$ \\
\hline \multirow[t]{3}{*}{$\begin{array}{l}\text { WBC } \\
\left(10^{6} / \mathrm{L}\right)\end{array}$} & Control & $\begin{array}{l}8233 \pm \\
208^{\mathrm{a} 1}\end{array}$ & $\begin{array}{l}7517 \pm \\
104^{\mathrm{b} 1}\end{array}$ & $\begin{array}{l}7967 \pm \\
651^{\mathrm{b} 1}\end{array}$ & $\begin{array}{l}8133 \pm \\
208^{b 1}\end{array}$ & $\begin{array}{l}7930 \pm \\
451^{\mathrm{b} 1}\end{array}$ \\
\hline & 1.06 & $\begin{array}{l}8667 \pm \\
115^{\mathrm{a} 1}\end{array}$ & $\begin{array}{l}10867 \pm \\
651^{\mathrm{a} 2}\end{array}$ & $\begin{array}{l}12200 \pm \\
361^{\mathrm{a} 2}\end{array}$ & $\begin{array}{l}13233 \pm \\
404^{\mathrm{a} 2}\end{array}$ & $\begin{array}{l}9233 \pm \\
321^{\mathrm{a} 1}\end{array}$ \\
\hline & 2.11 & $\begin{array}{l}8567 \pm \\
321^{\mathrm{a} 1}\end{array}$ & $\begin{array}{l}12100 \pm \\
300^{\mathrm{a} 2}\end{array}$ & $\begin{array}{l}12633 \pm \\
208^{\mathrm{a} 2}\end{array}$ & $\begin{array}{l}12600 \pm \\
361^{\mathrm{a} 2}\end{array}$ & $\begin{array}{l}9400 \pm \\
100^{\mathrm{a} 1}\end{array}$ \\
\hline
\end{tabular}




\begin{tabular}{|c|c|c|c|c|c|c|}
\hline \multirow[t]{2}{*}{ Parameter } & \multirow{2}{*}{$\begin{array}{l}\text { Conc. } \\
\text { (mg/L) }\end{array}$} & \multicolumn{5}{|c|}{ Duration (Day) } \\
\hline & & 1 & 5 & 10 & 15 & 5-d with. \\
\hline & 5.28 & $\begin{array}{l}8533 \pm \\
208^{\mathrm{a} 1}\end{array}$ & $\begin{array}{l}10933 \pm \\
702^{\mathrm{a} 2}\end{array}$ & $\begin{array}{l}13650 \pm \\
250^{\mathrm{a} 2}\end{array}$ & $\begin{array}{l}12733 \pm \\
153^{\mathrm{a} 2}\end{array}$ & $\begin{array}{l}10233 \pm \\
153^{a 12}\end{array}$ \\
\hline \multicolumn{7}{|c|}{$\begin{array}{l}\text { Values with different alphabet superscript along a column for each parameter was significantly } \\
\text { different, while values with different numeric superscript across a row were significantly different ( } p \\
0.05 \text { ). }\end{array}$} \\
\hline
\end{tabular}

The hematological derivatives $\mathrm{MCV}, \mathrm{MCH}$ and $\mathrm{MCHC}$ did not show any obvious change due to exposure of $C$. gariepinus to CPZ (supplementary Table S2). Change in MCV was very weakly significantly different overall between the groups $\left(F_{3,40}=4.261, p=0.011, \eta_{p}{ }^{2}=0.242\right)$, but the effect size was $\sim 25.0 \%$ and cannot be said to be due to the drug. The source of the significant difference was not obvious from LSD before and after Bonferroni adjustment. Similarly, the source of a weak significant difference between the $\mathrm{MCH}$ of the groups $\left(\mathrm{F}_{3,40}=5.127, \mathrm{p}=0.004, \eta_{\mathrm{p}}{ }^{2}=0.278\right)$, was not obvious from multiple comparisons. The values of $\mathrm{MCHC}$ also did not differ in the control and the exposed groups.

\section{Biochemical changes in C. gariepinus exposed to CPZ}

The activities of aspartate aminotransferase (AST), alanine aminotransferase (ALT) and alkaline phosphatase (ALP) were significantly affected by the 15-day exposure of $C$. gariepinus to CPZ (Table 3 ). AST (concentration: $F_{3,40}=141.934, p<0.0001, \eta_{p}{ }^{2}=0.914$; duration: $F_{4,40}=184.371, p<0.0001, \eta_{p}{ }^{2}=$ 0.949), ALT (concentration: $F_{3,40}=78.081, p<0.0001, \eta_{p}^{2}=0.854$; duration: $F_{3,40}=110.624, p<0.0001$, $\eta_{p}{ }^{2}=0.917$ ) and ALP (concentration: $F_{3,40}=176.100, p<0.0001, \eta_{p}{ }^{2}=0.930$; duration: $F_{4,40}=93.178, p<$ $\left.0.0001, \eta_{p}{ }^{2}=0.903\right)$ activities were increased significantly with increasing CPZ concentration and exposure duration. The effect of $\mathrm{CPZ}$ on all three parameters was significantly enhanced by prolongation of exposure $\left(p<0.0001, \eta_{p}{ }^{2} \sim 0.800\right)$. The effect of CPZ on AST, ALT and ALP activity waned on 5-day withdrawal of the fish from the drug. 
Table 3

Changes in blood aspartate aminotransferase (AST), alanine aminotransferase (ALT) and alkaline phosphatase (ALP) on 15-day exposure of juvenile Clarias gariepinus to CBZ

\begin{tabular}{|c|c|c|c|c|c|c|}
\hline \multirow[t]{2}{*}{ Parameter } & \multirow{2}{*}{$\begin{array}{l}\text { Conc. } \\
\text { (mg/L) }\end{array}$} & \multicolumn{5}{|c|}{ Duration (Day) } \\
\hline & & 1 & 5 & 10 & 15 & $\begin{array}{l}\text { 5-d } \\
\text { withdrawal }\end{array}$ \\
\hline \multirow[t]{4}{*}{ AST (U/L) } & Control & $\begin{array}{l}21.67 \pm \\
1.53^{\mathrm{a} 1}\end{array}$ & $\begin{array}{l}23.67 \pm \\
1.16^{\mathrm{b} 1}\end{array}$ & $\begin{array}{l}22.33 \pm \\
1.53^{\mathrm{b} 1}\end{array}$ & $\begin{array}{l}21.33 \pm \\
2.31^{\mathrm{b} 1}\end{array}$ & $\begin{array}{l}23.33 \pm \\
4.04^{\mathrm{a} 1}\end{array}$ \\
\hline & 1.06 & $\begin{array}{l}20.33 \pm \\
1.16^{\mathrm{a} 1}\end{array}$ & $\begin{array}{l}33.67 \pm \\
1.53^{\mathrm{a} 2}\end{array}$ & $\begin{array}{l}34.67 \pm \\
0.58^{\mathrm{a} 2}\end{array}$ & $\begin{array}{l}37.67 \pm \\
0.58^{\mathrm{a} 2}\end{array}$ & $\begin{array}{l}24.67 \pm \\
0.58^{\mathrm{a} 1}\end{array}$ \\
\hline & 2.11 & $\begin{array}{l}22.33 \pm \\
2.52^{\mathrm{a} 1}\end{array}$ & $\begin{array}{l}35.00 \pm \\
1.00^{\mathrm{a} 2}\end{array}$ & $\begin{array}{l}30.67 \pm \\
1.53^{\mathrm{a} 2}\end{array}$ & $\begin{array}{l}40.00 \pm \\
1.00^{\mathrm{a} 2}\end{array}$ & $\begin{array}{l}24.67 \pm \\
1.53^{\mathrm{a} 1}\end{array}$ \\
\hline & 5.28 & $\begin{array}{l}20.33 \pm \\
2.08^{\mathrm{a} 1}\end{array}$ & $\begin{array}{l}39.00 \pm \\
1.00^{\mathrm{a} 2}\end{array}$ & $\begin{array}{l}43.33 \pm \\
1.15^{\mathrm{a} 2}\end{array}$ & $\begin{array}{l}44.00 \pm \\
2.00^{\mathrm{a} 2}\end{array}$ & $\begin{array}{l}25.00 \pm \\
1.00^{\mathrm{a} 1}\end{array}$ \\
\hline \multirow[t]{4}{*}{ ALT (U/L) } & Control & $\begin{array}{l}31.67 \pm \\
3.22^{\mathrm{a} 1}\end{array}$ & $\begin{array}{l}33.00 \pm \\
2.65^{\mathrm{c} 1}\end{array}$ & $\begin{array}{l}32.33 \pm \\
3.06^{\mathrm{c} 1}\end{array}$ & $\begin{array}{l}32.00 \pm \\
5.30^{\mathrm{b} 1}\end{array}$ & $\begin{array}{l}30.67 \pm \\
3.22^{\mathrm{a} 1}\end{array}$ \\
\hline & 1.06 & $\begin{array}{l}33.00 \pm \\
2.65^{\mathrm{a} 1}\end{array}$ & $\begin{array}{l}37.67 \pm \\
1.53^{\mathrm{b} 2}\end{array}$ & $\begin{array}{l}47.00 \pm \\
3.00^{\mathrm{b} 3}\end{array}$ & $\begin{array}{l}52.33 \pm \\
2.08^{\mathrm{a} 4}\end{array}$ & $\begin{array}{l}34.67 \pm \\
0.58^{\mathrm{a} 1}\end{array}$ \\
\hline & 2.11 & $\begin{array}{l}31.33 \pm \\
1.16^{\mathrm{a} 1}\end{array}$ & $\begin{array}{l}41.33 \pm \\
1.16^{\mathrm{a} 2}\end{array}$ & $\begin{array}{l}52.00 \pm \\
4.00^{\mathrm{a} 3}\end{array}$ & $\begin{array}{l}56.00 \pm \\
1.00^{\mathrm{a} 3}\end{array}$ & $\begin{array}{l}36.67 \pm \\
1.16^{\mathrm{a} 1}\end{array}$ \\
\hline & 5.28 & $\begin{array}{l}31.33 \pm \\
2.08^{\mathrm{a} 1}\end{array}$ & $\begin{array}{l}43.33 \pm \\
1.16^{\mathrm{a} 2}\end{array}$ & $\begin{array}{l}56.67 \pm \\
1.53^{\mathrm{a} 3}\end{array}$ & $\begin{array}{l}52.67 \pm \\
0.58^{\mathrm{a} 3}\end{array}$ & $\begin{array}{l}35.00 \pm \\
1.00^{\mathrm{a} 1}\end{array}$ \\
\hline \multirow[t]{4}{*}{ ALP (U/L) } & Control & $\begin{array}{l}41.33 \pm \\
3.06^{\mathrm{a} 1}\end{array}$ & $\begin{array}{l}35.67 \pm \\
1.53^{\mathrm{c} 1}\end{array}$ & $\begin{array}{l}41.33 \pm \\
1.16^{\mathrm{b} 1}\end{array}$ & $\begin{array}{l}41.33 \pm \\
2.31^{\mathrm{b} 1}\end{array}$ & $\begin{array}{l}42.67 \pm \\
3.51^{\mathrm{a} 1}\end{array}$ \\
\hline & 1.06 & $\begin{array}{l}39.67 \pm \\
0.58^{\mathrm{a} 1}\end{array}$ & $\begin{array}{l}49.00 \pm \\
2.65^{\mathrm{b} 2}\end{array}$ & $\begin{array}{l}54.67 \pm \\
3.06^{\mathrm{a} 2}\end{array}$ & $\begin{array}{l}59.33 \pm \\
1.16^{\mathrm{a} 2}\end{array}$ & $\begin{array}{l}48.33 \pm \\
2.08^{\mathrm{a} 2}\end{array}$ \\
\hline & 2.11 & $\begin{array}{l}44.00 \pm \\
2.00^{\mathrm{a} 1}\end{array}$ & $\begin{array}{l}58.00 \pm \\
1.73^{\mathrm{a} 2}\end{array}$ & $\begin{array}{l}59.00 \pm \\
1.74^{\mathrm{a} 2}\end{array}$ & $\begin{array}{l}60.00 \pm \\
1.73^{\mathrm{a} 2}\end{array}$ & $\begin{array}{l}53.33 \pm \\
1.16^{\mathrm{a} 2}\end{array}$ \\
\hline & 5.28 & $\begin{array}{l}42.00 \pm \\
1.73^{\mathrm{a} 1}\end{array}$ & $\begin{array}{l}57.33 \pm \\
1.16^{\mathrm{a} 2}\end{array}$ & $\begin{array}{l}59.67 \pm \\
0.58^{\mathrm{a} 2}\end{array}$ & $\begin{array}{l}63.33 \pm \\
1.16^{\mathrm{a} 2}\end{array}$ & $\begin{array}{l}50.33 \pm \\
1.53^{\mathrm{a} 2}\end{array}$ \\
\hline $\begin{array}{l}\text { Values with } \\
\text { different, w } \\
0.05 \text { ). }\end{array}$ & $\begin{array}{l}\text { fferent a } \\
\text { e values }\end{array}$ & $\begin{array}{l}\text { et supe } \\
\text { ifferent }\end{array}$ & $\begin{array}{l}\text { long a c } \\
\text { c supers }\end{array}$ & $\begin{array}{l}\text { for each } \\
\text { cross a r }\end{array}$ & $\begin{array}{l}\text { neter was } \\
\text { e signifis }\end{array}$ & $\begin{array}{l}\text { ficantly } \\
\text { different ( } p<<\end{array}$ \\
\hline
\end{tabular}

Exposure of $C$. gariepinus to $\mathrm{CPZ}$ resulted in reduction of protein in a duration-dependent manner (Fig. 1). Significant reduction in serum protein occurred on days 5,10 and 15 of exposure in all drug 
concentrations $\left(F_{3,40}=33.779, p<0.0001, \eta_{p}{ }^{2}=0.717\right)$. The magnitude of hyperproteinemia associated with the drug exposure was similar from days 5 to 15 , and normalcy returned on withdrawal of the fish from the drug. CPZ exposure was associated with significant reduction in serum glucose concentration $\left(F_{3,40}=109.525, p<0.0001, \eta_{p}{ }^{2}=0.891\right)$. Though the glucose concentration was significantly elevated during the exposure period $\left(F_{4,40}=58.776, p<0.0001, \eta_{p}{ }^{2}=0.855\right)$, the concentration in exposed fish was not different between day-5 and day-15. Glucose concentration returned to same level as control on 5-day withdrawal of the fish (Fig. 2).

\section{Changes In Morphological Parameters}

The changes in $\mathrm{CF}$ and $\mathrm{HSI}$ in the fish exposed to $\mathrm{CPZ}$ are summarized in Table 4. Although there were concentration and duration dependent variations in the CF, the LSD test showed no significant differences $(p>0.05)$. HSI of the fish were different between the groups $\left(F_{3,40}=4.956, p=0.005, \eta_{p}{ }^{2}=0.271\right)$, though the effect size was $27.1 \%$. A significant increase in HSI occurred on day-15 in fish at $5.28 \mathrm{mg} / \mathrm{L} \mathrm{CPZ}$, and explain the significant interaction effect $\left(F_{12,40}=3.813, p=0.001, \eta_{p}^{2}=0.534\right)$. 
Table 4

Changes in condition factor (CF) and hepatosomatic indices (HSI) of juvenile Clarias gariepinus on 15day exposure to $\mathrm{CBZ}$

\begin{tabular}{|c|c|c|c|c|c|c|}
\hline \multirow[t]{2}{*}{ Parameter } & \multirow{2}{*}{$\begin{array}{l}\text { Conc. } \\
\text { (mg/L) }\end{array}$} & \multicolumn{5}{|c|}{ Duration (Day) } \\
\hline & & 1 & 5 & 10 & 15 & $\begin{array}{l}\text { 5-d } \\
\text { withdrawal }\end{array}$ \\
\hline \multirow[t]{4}{*}{$\mathrm{CF}$} & Control & $\begin{array}{l}0.46 \pm \\
0.03^{\mathrm{a} 1}\end{array}$ & $\begin{array}{l}0.68 \pm \\
0.11^{\mathrm{a} 1}\end{array}$ & $\begin{array}{l}0.60 \pm \\
0.09^{\mathrm{a} 1}\end{array}$ & $\begin{array}{l}0.53 \pm \\
0.05^{\mathrm{a} 1}\end{array}$ & $0.51 \pm 0.07^{\mathrm{a} 1}$ \\
\hline & 1.06 & $\begin{array}{l}0.61 \pm \\
0.15^{\mathrm{a} 1}\end{array}$ & $\begin{array}{l}0.71 \pm \\
0.08^{\mathrm{a} 1}\end{array}$ & $\begin{array}{l}0.57 \pm \\
0.13^{\mathrm{a} 1}\end{array}$ & $\begin{array}{l}0.52 \pm \\
0.09^{\mathrm{a} 1}\end{array}$ & $0.60 \pm 0.08^{\mathrm{a} 1}$ \\
\hline & 2.11 & $\begin{array}{l}0.61 \pm \\
0.13^{\mathrm{a} 1}\end{array}$ & $\begin{array}{l}0.65 \pm \\
0.07^{\mathrm{a} 1}\end{array}$ & $\begin{array}{l}0.67 \pm \\
0.01^{\mathrm{a} 1}\end{array}$ & $\begin{array}{l}0.70 \pm \\
0.03^{\mathrm{a} 1}\end{array}$ & $0.54 \pm 0.03^{\mathrm{a} 1}$ \\
\hline & 5.28 & $\begin{array}{l}0.63 \pm \\
0.15^{\mathrm{a} 1}\end{array}$ & $\begin{array}{l}0.57 \pm \\
0.10^{\mathrm{a} 1}\end{array}$ & $\begin{array}{l}0.55 \pm \\
0.09^{\mathrm{a} 1}\end{array}$ & $\begin{array}{l}0.46 \pm \\
0.03^{\mathrm{a} 1}\end{array}$ & $0.51 \pm 0.00^{\mathrm{a} 1}$ \\
\hline \multirow[t]{4}{*}{$\mathrm{HSI}$} & Control & $\begin{array}{l}1.69 \pm \\
0.02^{\mathrm{a} 1}\end{array}$ & $\begin{array}{l}1.73 \pm \\
0.02^{\mathrm{a} 1}\end{array}$ & $\begin{array}{l}1.73 \pm \\
1.15^{\mathrm{a} 1}\end{array}$ & $\begin{array}{l}1.50 \pm \\
0.33^{b 1}\end{array}$ & $1.98 \pm 0.18^{\mathrm{a} 1}$ \\
\hline & 1.06 & $\begin{array}{l}1.37 \pm \\
0.23^{\mathrm{a} 1}\end{array}$ & $\begin{array}{l}1.70 \pm \\
0.50^{\mathrm{a} 1}\end{array}$ & $\begin{array}{l}1.56 \pm \\
0.48^{\mathrm{a} 1}\end{array}$ & $\begin{array}{l}0.99 \pm \\
0.08^{b 1}\end{array}$ & $1.97 \pm 0.09^{\mathrm{a} 1}$ \\
\hline & 2.11 & $\begin{array}{l}1.67 \pm \\
0.18^{\mathrm{a} 1}\end{array}$ & $\begin{array}{l}1.70 \pm \\
0.35^{\mathrm{a} 1}\end{array}$ & $\begin{array}{l}1.37 \pm \\
0.05^{\mathrm{a} 1}\end{array}$ & $\begin{array}{l}1.20 \pm \\
0.09^{\mathrm{b} 1}\end{array}$ & $1.86 \pm 0.00^{\mathrm{a} 1}$ \\
\hline & 5.28 & $\begin{array}{l}1.96 \pm \\
0.05^{\mathrm{a} 1}\end{array}$ & $\begin{array}{l}1.69 \pm \\
0.24^{\mathrm{a} 1}\end{array}$ & $\begin{array}{l}1.43 \pm \\
0.20^{\mathrm{a} 1}\end{array}$ & $\begin{array}{l}2.84 \pm \\
0.25^{\mathrm{a} 2}\end{array}$ & $1.84 \pm 0.09^{\mathrm{a} 1}$ \\
\hline
\end{tabular}

\section{Discussion}

Exposure of juvenile $C$. gariepinus to $\mathrm{CPZ}$ elicited behavioral changes such as abnormal mucus secretion, skin coloration and air gulping which were more pronounced at higher CPZ concentrations. Other responses such as opercula movement and swimming were less pronounced with increasing CPZ concentrations. Similar behavioral responses were reported in Carassius auratus exposed to CPZ (Li et al., 2008), C. gariepinus exposed to chloramphenicol and CPZ (Nwani et al., 2014a; Atama et al., 2020) and Danio rerio exposed to tramadol (Bachour et al., 2020). Zang (2003) also reported behavioral abnormalities in animals after administration of small doses of CPZ. 
Blood parameters have been used in assessing the health condition of fish as they are the first target for xenobiotic action in the body (Melefa et al., 2020). Exposure of $C$. gariepinus to CPZ sublethal concentrations induced reduction in PCV, RBC and $\mathrm{Hb}$ values. The reduction in the values of these parameters may be due to the impairment of their synthesis by CPZ. Similar results have been reported in C. gariepinus exposed to drugs such as praziquantel (Nwani et al., 2016), ivermectin (Ogueji et al., 2019) and clotrimazole (Melefa et al., 2020). Our results indicate that CPZ provoked significant elevation of WBC in the peripheral blood of the exposed fish. The increased WBC levels may indicate immunoprotective activity elicited against the drug. The elevated WBC in the blood may also be due to the stimulation of the T-lymphocytes by CPZ (Campbell 1996). Similar increase in WBC were obtained in $C$. gariepinus exposed to chloramphenicol (Nwani et al., 2014a), diazepam (Ogueji et al, 2018), clotrimazole (Melefa et al., 2020). Ajima et al. (2017) also reported the elevation of WBC in verapamil-exposed Oreochromis niloticus. Hematological indices are useful in determining the type of anemia in organisms. In the present study, there were no significant difference in the values of $\mathrm{MCV}, \mathrm{MCH}$ and $\mathrm{MCHC}$ in exposed fish compared to the control. Similar to our finding, Nwani et al. (2014b) reported no significant difference in $\mathrm{MCV}, \mathrm{MCH}$ and $\mathrm{MCHC}$ values in C. gariepinus exposed to praziquantel. Li et al. (2011) also reported no significant change of $\mathrm{MCHC}$ in Onchorrynchus mykiss exposed to carbamazepine. Contrary to our report however, increased $\mathrm{MCV}, \mathrm{MCH}$ and $\mathrm{MCHC}$ values were reported in $C$. gariepinus exposed to clotrimazole (Melefa et al., 2020) and acetylsalicylic acid (Siddeswaran et al., 2020). The leucocyte differentials in $C$. gariepinus exposed to CPZ were comparable to the control throughout the duration of the experiment. Similar observations have been recorded in other fishes exposed to different toxicants (Velisek et al., 2009; Mohammad et al., 2012; Nwani et al., 2016).

CPZ associated concentration and duration dependent elevation in activities of AST, ALT and ALP occurred in $C$. gariepinus. The significant increase of these parameters in the exposed fish may be attributed to the hepatotoxic effects of CPZ. Our result is in agreement with the reports of other authors that recorded elevation of biochemical parameters in pharmaceutical-exposed fish (Owoade et al., 2019; Odo et al., 2020; Siddeswaran et al., 2020). There was significant decrease in the protein and glucose levels in C. gariepinus exposed to CPZ. The decrease in protein may be due to its increased use to make up for the high metabolic activities that may have induced stress and hepatic damage. The decrease in glucose level as observed in the present study may be due to possible impairment of the kidney which may have inhibited glucose biosynthesis as a result of damage to the liver cells. Further investigations are however needed to conclusively validate our report. The return of the protein and glucose to the control levels after the 5-day withdrawal from CPZ may indicate that the impairments were due to the drug.

Condition factor gives an indication of wellbeing of the fish in the environment. In the present study, CF showed no obvious response to the CPZ exposure. Similar to our report, Melefa et al. (2020) reported that $\mathrm{CF}$ was not altered in C. gariepinus exposed to clotrimazole. Further, there was no significant difference in CF in Oncorhynchus mykiss exposed to clotrimazole and carbamazepine (Li et al., 2011; Burkina et al., 2016). No significant change in CF was also reported in C. gariepinus exposed to praziquantel (Nwani et al., 2014b) and ivermectin (Odo et al., 2020). Our result thus imply that CPZ may have no major influence 
on the ratio of length to weight of the fish. Exposure of $C$. gariepinus to $\mathrm{CPZ}$ resulted in elevation of HSI which was significantly different from the control on day 15 at $5.28 \mathrm{mg} / \mathrm{L} \mathrm{CPZ}$. Changes in the HSI have also been reported in fish species exposed to pharmaceuticals and other substances (Sogbanmu et al., 2018; Odo et al., 2020).

\section{Conclusion}

The study indicates that $\mathrm{CPZ}$ elevates the values of WBC, PCV, RBC, decreased the level of $\mathrm{Hb}$ but the $\mathrm{MCV}, \mathrm{MCH}, \mathrm{MCHC}$ values and the white blood differentials were comparable to the control. There was also significant increase in the values of aspartate aminotransferase, alanine aminotransferase and alkaline phosphatase but a reduction in the values of protein and glucose. The values of condition factor and hepatosomatic index in the exposed fish showed mixed pattern. CPZ is toxic to $C$. gariepinus and its potential ecological risks to aquatic biota on a long term should be further investigated.

\section{Declarations}

Ethical approval: The fish were treated in accordance with the rules conforming to principles of laboratory animal care as obtained from the ethical committee on the use of experimental animals Faculty of Biological Science, University of Nigeria Nsukka (UNN-ECFBS-00234).

Consent to participate: Not applicable

Availability of data and materials: Not applicable.

Consent for publication: Not applicable.

Competing interest: The authors declare that there is no competing interest.

Funding: The research is self-sponsored and not funded by anybody or organizations.

Authors contributions: This work was carried out in collaboration among all authors. Martins Nnamdi Okpe and Ifeanyi Oscar Aguzie designed the study, performed the laboratory analysis. Chukwuebuka Christabel Eze and Martin Abdubala Okpanachi wrote the protocol and the first draft of the manuscript. Hope Chinwe Ezinwa and Christopher Didigwu Nwani managed the statistical analyses of the study. Uduak Aletan, Henrietta ljeoma Kelle and Maureen N Chukwu managed the literature searches and edited the final manuscript. All authors read and approved the final manuscript.

Acknowledgements: The authors are grateful to the staff of Department, Applied Biology and Biotechnology, Enugu State University of Science and Technology for their general support during the research. We are also grateful to the management of University of Nigeria for giving us access to their laboratory.

\section{References}


1. Ajima MNO, Pandey PK, Kumar K, Poojary N (2017) Assessment of mutagenic, hematological and oxidative stress biomarkers in liver of Nile tilapia, Oreochromis niloticus (Linnaeus, 1758) in response to sublethal verapamil exposure. Drug Chem Toxicol 40: 286-294.

2. Atama CA, Nnaji EC, Ezeoyili IC, Udeani FO, Onovo CJ, Ossai NI, Aguzie IO, Nwani CD (2020) Neuromodulatory and oxidative stress evaluations in African catfish Clarias gariepinus exposed to antipsychotic drug chlorpromazine, Drug Chem Toxicol. DOI: 10.1080/01480545.2020.1822391

3. American Public Health Association, (APHA) (2005). Standard Methods for the Examination of Water and Waste Water, 21st edn. APHA, Washington, DC.

4. Bachour RL, Golovko O, Kellner M, Pohl J (2020) Behavioral effects of citalopram, tramadol, and binary mixture in zebrafish (Danio rerio) larvae. Chemosphere 238:124587

5. Campbell TW (1996) In: Mader DR (Ed.), Clinical Pathology. Reptile Medicine and Surgery. W.B. Saunders Company, Philadelphia, PA, pp. 248-257.

6. Dacie JV, Lewis SM (1984) Practical hematology. 6th ed. New York, London: Churchill.

7. Ebele AJ, Oluseyi T, Drage DS, Harrad S, Abdallah MAE (2020) Occurrence, seasonal variation and human exposure to pharmaceuticals and personal care pro- ducts in surface water, groundwater and drinking water in Lagos State, Nigeria. Emerg Contam 6: 124-132.

8. Fernandez C, Gonza'lez-Doncel M, Pro J, Carbonell G, Tarazona JV (2010) Occurrence of pharmaceutically active compounds in surface waters of the Henares- Jarama-Tajo River system (Madrid, Spain) and a potential risk characterization. Sci Total Environ 3:543-51.

9. Hinfray N, Baudiffier D, Leal MC, Porcher JM, Aït-Aïssa S, Le Gac F, Schulz RW, Brion F (2011) Characterization of testicular expression of P450 17a-hydroxylase, 17,20-lyase in zebrafish and its perturbation by the pharmaceutical fungicide clotrimazole. Gen. Comp. Endocrinol 174: 309-317.

10. Li T, Zhou Q, Zhang N, Luo Y (2008) Toxic effects of chlorpromazine on Carassius auratus and its oxidative stress. J Environ Sci Health. Part. B, Pest Food Contam Agric Wastes 43: 638-643.

11. Li ZH, Velisek J, Zlabek V, Grabic R, Machova J, Kolarovad J, Li P, Randak T (2011) Chronic toxicity of verapamil on juvenile rainbow trout (Onchohynchus mykiss). Effects on morphological indices, hematological parameters and antioxidant responses. J Hazard Mater 185: 870-880.

12. Melefa TD, Mgbenka BO, Aguzie IO, Andong FA Nwakor U, Nwani CD (2020) Morphological, hematological and biochemical changes in African catfish Clarias gariepinus (Burchell 1822) juveniles exposed to clotrimazole. Comp Biochem Physiol, Part C 236:108815.

13. Mohammad NSM, Soltani M, Kamali A, Imanpoor MR, Sharifpour I, Khara H (2012) Effects of organophosphate, diazinon on some hematological and bio-chemical changes in Rutilus frisii kutum (Kamensky, 1901) male brood stocks. Iran J Fish Sci 11: 105-117.

14. Nwani CD, Mkpadobi BN, Onyishi GC, Echi PC, Chukwuka CO, Oluah SN, Ivoke N (2014a) Changes in behavior and hematological parameters of freshwater African Catfish Clarias gariepinus (Burchell 1822) following sublethal exposure to chloramphenicol. Drug Chem Toxicol 37: 107-113.

15. Nwani CD, Nnaji CN, Oluah SN, Echi PC, Nwamba HO, Ikwuagwu OE, Ajima MNO (2014b) Mutagenic and physiological responses in the juveniles of African catfish, Clarias gariepinus (Burchell 1822) 
following short term exposure to praziquantel. Tissue and Cell 46: 264-273.

16. Nwani CD, Somdare PO, Ukonze JA, Ejere VC, Nwadinigwe AO, Nwani JC, Odo GE, Ugbor NO (2016) Subchronic exposure to fenthion induces hematological changes in liver tissue of African catfish Clarias gariepinus. J Aquat Animal Health 28: 229 -234.

17. Ochei U, Kolhatkar $O$ (2008) Colorimetric method for the determination of serum glutamic oxaloacetic and glutamic pyruvic transaminase. American J Clin Pathol 766: 28-56.

18. Odo UU, Ezeoyili Cl, Aguzie IO, Oluah NS, Madu JC, Nwani CD (2020) Effect of ivermectin on biometric characteristics and organ biomarkers of African catfish Clarias gariepinus. Mar Freshw Behav Physiol 53: 17-33.

19. Ogueji EO, Nwani CD, Iheanacho SC, Mbah CE, Ibrahim BU (2018) Hematological alterations in the African catfish (Clarias gariepinus) juveniles exposed to sub-chronic concentrations of diazepam. Nigerian J Fish 14: 1170-1177.

20. Ogueji EO, Nwani CD, Mbah MC, Nweke FN (2019) Acute hematological toxicity of ivermectin to juvenile Clarias gariepinus. Toxicol Environ Chem 101: 300 - 314.

21. Oleveira LLD, Antunes SC, Gonclaves F, Rocha O, Nunes B (2016) Acute and chronic ecotoxicological effects of four pharmaceutical drugs on Cladocera Daphnia magna. Drug Chem Toxicol 39: 13-21.

22. Owoade AO, Adetutu A, Olorunnisola OS (2019) Hematological and biochemical changes in blood, liver and kidney tissues under the effect of tramadol treatment. J Alcohol Drug Depend 7: 327.

23. Porsbring T, Blanck H, Tjellström H, Backhaus $T$ (2008) Toxicity of the pharmaceutical clotrimazole to marine microalgal communities. Aquat Toxicol 91: 203-211.

24. Reitman S, Frankel S (1957) A colorimetric method for the determination of serum glutamic oxalo acetic and glutamic pyruvic transaminase. American J Clin Pathol 766: 28-56.

25. Siddeswaran S, Umamaheswari S, Ramesh M (2020) Toxicity assessment of acetylsalicylic acid to freshwater fish Cyprinus carpio: Hematological, biochemical, enzymological and antioxidant response. In: L.M Gomez (ed.). Non-steroidal anti-inflammatory Siddeswaran et al., 2020) drugs in water: emerging contaminants and ecological impact. Hdb Enochem, https://doi.org/10.1007/098_2020_549.

26. Sogbanmu TO, Osibona AO, Oguntunde OA, Otitoloju AA (2018) Biomarkers of toxicity in Clarias gariepinus exposed to sublethal concentration of polycyclic aromatic hydrocarbons. Afr J Aquat Sci 43: 281-292.

27. Suzuki H, Gen K, Inoue $Y$ (2013) Comparison of the anti-dopamine $D \otimes$ and anti-serotonin 5-HT(2A) activities of chlorpromazine, bromperidol, haloperidol and second-generation antipsychotics parent compounds and metabolites thereof. J Psychopharmacol 27: 396-400.

28. Velisek J, Stesjskal V, Kouril J, Svobodovo Z (2009) Comparison of the effects of four anesthetic on biochemical blood profiles of Perch. Aqualcult Res 40: 354-361.

29. Wassmur B, Grans J, Norstrom E, Wallin M, Celander MC (2013) Interactions of pharmaceuticals and other xenobiotics on key detoxification mechanisms and cytoskeleton in Poeciliopsis lucida hepatocellular carcinoma, PLHC-1 cell line. Toxicol in Vitro 27: 111-120. 
30. White A, Fletcher TC (1985) Seasonal changes in serum glucose and condition of the plaice, Pleuronectes platessa L. J Fish Biol 26: 755-764.

31. Yuan S, Jiang X, Xia X, Zhang H, Zheng S (2013) Detection, occurrence and fate of psychiatric pharmaceuticals in psychiatric hospital and municipal wastewater treatment plants in Beijing, China. Chemosphere 10: 2520-2525.

32. Zhang, $\mathrm{H}$ (2003) The harmful residue of drugs in animal products and controlling methods. Guizhou Anim Sci Vet Med 27, 6-9.

\section{Figures}

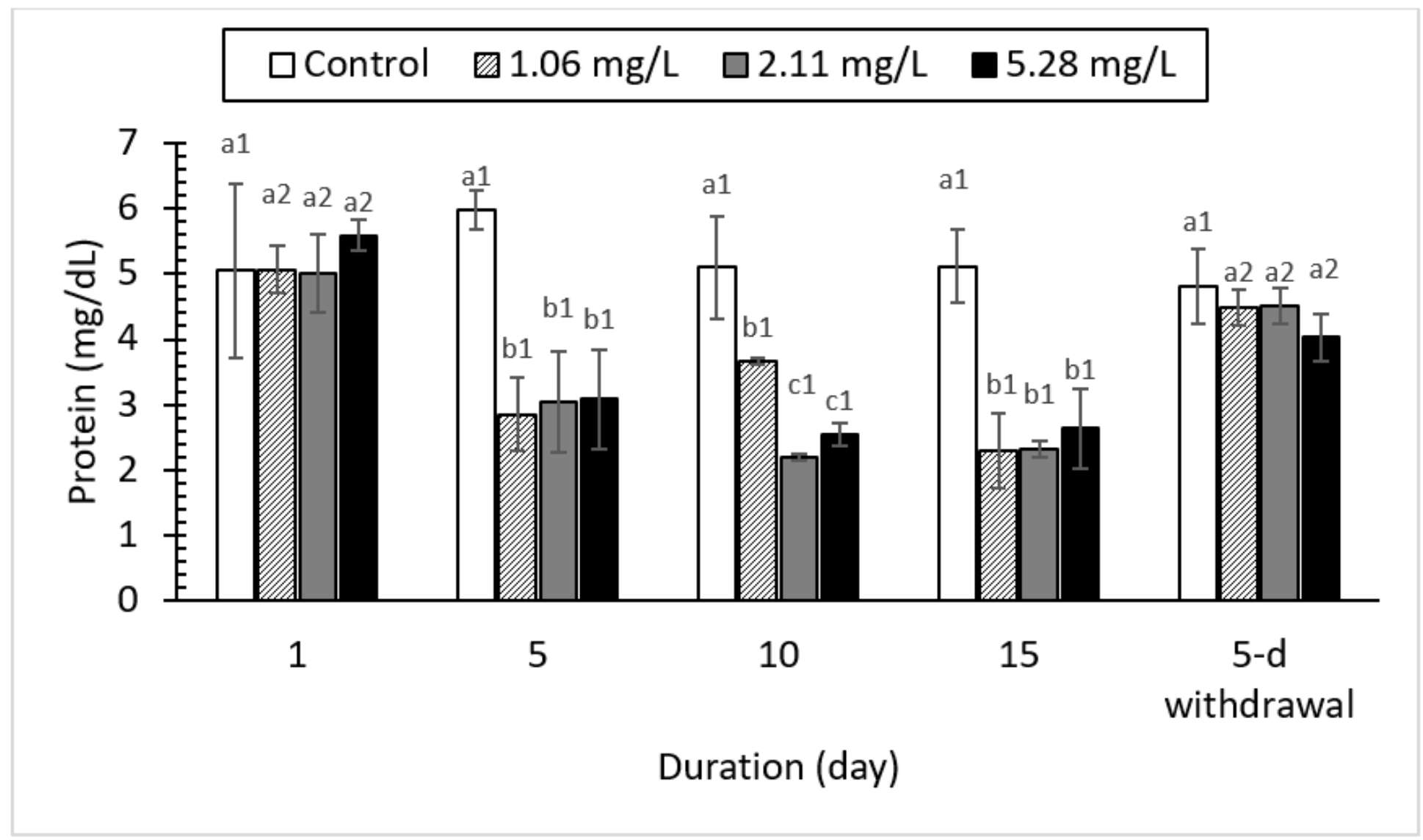

Figure 1

Changes in serum total protein concentration on exposure of Clarias gariepinus to CBZ. Bars with different alphabet label were significantly different for a given day, while bars with different numeric labels were significantly different between durations for a given CBZ concentration $(p<0.05)$. 


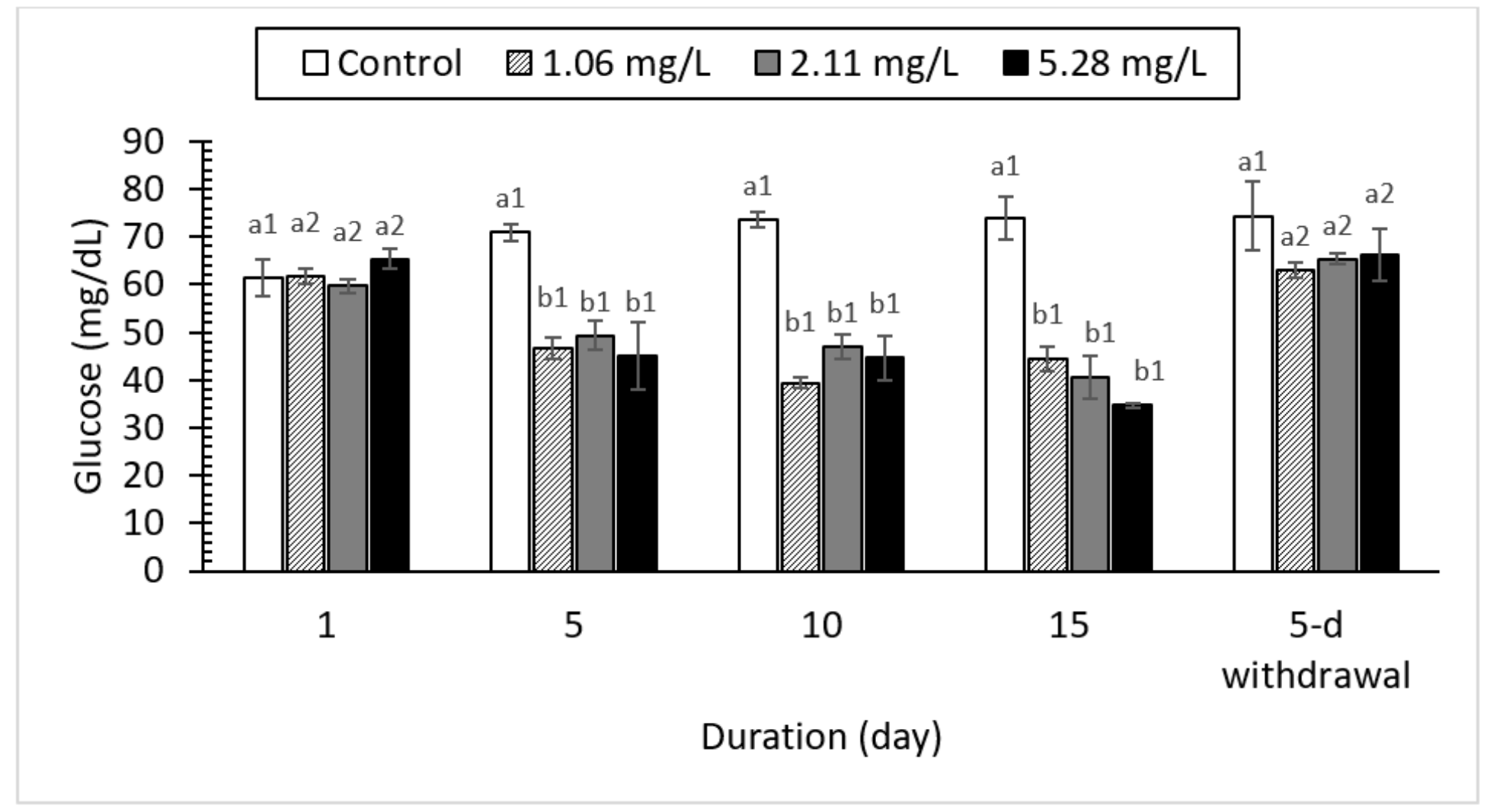

Figure 2

Changes in serum glucose concentration on exposure of Clarias gariepinus to CBZ. Bars with different alphabet label were significantly different for a given day, while bars with different numeric labels were significantly different between durations for a given CBZ concentration $(p<0.05)$.

\section{Supplementary Files}

This is a list of supplementary files associated with this preprint. Click to download.

- TableS1Whiteblooddifferentials.docx

- TableS2MCHMCHC.docx 